\title{
Reduced expression of shaggy, the gene encoding protein kinase GSK3, in dopaminergic neurons increases Drosophila melanogaster lifespan
}

\author{
E.R. Veselkina ${ }^{1 *}$, M.V. Trostnikov ${ }^{1,2}$, A.V. Krementsova ${ }^{1,3}$, N.V. Roshina ${ }^{1,2}$, \\ E.G. Pasyukova ${ }^{1}$ \\ ${ }^{1}$ Institute of Molecular Genetics of RAS, Moscow, Russia \\ ${ }^{2}$ Vavilov Institute of General Genetics of RAS, Moscow, Russia \\ ${ }^{3}$ Emmanuel Institute of Biochemical Physics of RAS, Moscow, Russia \\ *e-mail:veselkinaer@gmail.com
}

Key words: life span, protein kinase, GSK3, the nervous system, dopaminergic neurons, Drosophila

Motivation and Aim: Phosphorylation cascades play crucial roles in systemic regulation of numerous metabolic processes. Various protein kinases are well recognized as basic participants of these cascades. A serine-threonine protein kinase GSK3 (glycogen synthase kinase-3) is a well-known protein involved in many functionally different signaling pathways and metabolic processes. In particular, it is important for neurogenesis and neuronal function. We have previously shown that several genes involved in asymmetric neuroblast division, including shaggy ( $\mathrm{sgg}$ ) encoding Drosophila melanogaster GSK3, affect Drosophila lifespan. In order to better understand the role of GSK3 in the control of aging and neuronal function, we assessed the effects of tissue-specific increase and decrease in GSK3 expression on lifespan. Our results demonstrated that differential expression of GSK3 is one of the mechanisms involved in a complex regulation of lifespan and aging.

To get closer to understanding molecular mechanisms underlying tissue-specific effects of GSK3 on lifespan, we studied effects of shaggy misexpression in dopaminergic neurons in more detail, using genetic approaches combined with RT-qPCR and immunohistochemical analyses.

Results: Dopaminergic neurons were the most sensitive to shaggy function: strong shaggy overexpression was lethal, moderate shaggy overexpression decreased male and female lifespan, while moderate decrease in GSK3 amounts increased mean female lifespan by $20 \%$ and reduced mean male lifespan by $20 \%$. The results were highly reproducible over several years. Different techniques (different Drosophila driver lines) were also used to double check the impact of reduces GSK3 amounts on female lifespan; positive effects were confirmed. Analysis of effects of $s g g$ misexpression in dopaminergic neurons on lifespan of mated flies is currently under way. We expect that these data will shed light on the role of fly physiology, sex and mating status in shaping GSK3 effects on lifespan. Moderate shaggy overexpression caused a severe reduction of the number of dopaminergic neurons in the brains of adult flies. Of note, decrease in the amount of dopaminergic neurons in the brain is an indication of a number of neurodegenerative diseases in humans, and analysis of molecular mechanisms involved in maintenance of the proper neuron amount and their integrity is of significant interest.

Conclusion: Alterations in the level of expression of GSK3 in dopaminergic neurons underlie systemic sex-specific changes in molecular mechanisms involved in a complex regulation of lifespan and aging.

Acknowledgements: Supported by the RFBR grant No. 18-34-00934 мол_а. 